\title{
Chaos in a Financial System with Fractional Order and Its Control via Sliding Mode
}

\author{
Paul Yaovi Dousseh, ${ }^{1}$ Cyrille Ainamon, ${ }^{1}$ Clément Hodévèwan Miwadinou $\mathbb{C}^{1,2,3}$ \\ Adjimon Vincent Monwanou, ${ }^{1}$ and Jean Bio Chabi Orou ${ }^{1}$ \\ ${ }^{1}$ Laboratoire de Mécaniques des fluides, de la Dynamique Non-linéaire et de la Modélisation des Systèmes Biologiques \\ (LMFDNMSB), Institut de Mathématiques et de Sciences Physiques (IMSP), Porto-Novo, Benin \\ ${ }^{2}$ Département de Physique, ENS-Natitingou, Université des Sciences, Technologies, Ingénierie et Mathématiques (UNSTIM), \\ Abomey, Benin \\ ${ }^{3}$ Laboratoire de Physique et Applications du Centre Universitaire de Natitingou, \\ Université Nationale des Sciences, Technologiques, Ingénierie et Mathématiques (UNSTIM), Abomey, Benin
}

Correspondence should be addressed to Clément Hodévèwan Miwadinou; clement.miwadinou@imsp-uac.org

Received 15 April 2021; Revised 26 May 2021; Accepted 3 July 2021; Published 19 July 2021

Academic Editor: Guillermo Huerta Cuellar

Copyright (c) 2021 Paul Yaovi Dousseh et al. This is an open access article distributed under the Creative Commons Attribution License, which permits unrestricted use, distribution, and reproduction in any medium, provided the original work is properly cited.

\begin{abstract}
In this paper, the dynamical behaviors and chaos control of a fractional-order financial system are discussed. The lowest fractional order found from which the system generates chaos is 2.49 for the commensurate order case and 2.13 for the incommensurate order case. Also, period-doubling route to chaos was found in this system. The results of this study were validated by the existence of a positive Lyapunov exponent. Besides, in order to control chaos in this fractional-order financial system with uncertain dynamics, a sliding mode controller is derived. The proposed controller stabilizes the commensurate and incommensurate fractional-order systems. Numerical simulations are carried out to verify the analytical results.
\end{abstract}

\section{Introduction}

Investigating chaos in dynamical systems is one of the most interesting topics which has been carried out extensively in different scientific fields such as medicine [1], biology [2], mathematics [3], and many others. In the literature, several dynamical systems presenting chaotic behaviors have been proposed such as the Lorenz system [4], the Chen system [5], the Lü system [6], and the Newton-Leipnik system [7]. During the last decades, many researchers have taken a great interest in the subject of chaotic systems' control. In [8], the authors used the sliding mode controller to eliminate chaos in a new uncertain chaotic dynamical system (Liu system). In [9], a robust adaptive sliding mode controller is used to remove chaos in a novel class of chaotic systems. Chaos control in the Lorenz, Chen, and Lü systems using the backstepping technique is performed in [10]. Chaos control in the
Newton-Leipnik system is carried out using linear feedback controllers in [11].

Fractional calculus involving fractional order derivatives, i.e., derivatives of noninteger order, has a history of over 300 years [12]. During those years, this theory was considered a purely mathematical concept. Recently, fractional order derivatives have been widely applied to several systems in many areas of research to better understand these systems [13-19]. Indeed, fractional order derivatives, possessing memory, can describe more accurately different nonlinear phenomena than integer order derivatives $[20,21]$. Very recently, some interesting developments and results in the theory and applications of fractional calculus have been obtained in the literature. For example, see [22], in which authors used Lie symmetry analysis to obtain an exact solution of the conformable heat equation. In [23], useful properties of the Lie group method with the invariance subspace method are combined to obtain a large family of 
exact solutions for the fractional Black-Scholes equation. Also, in [24], the necessary optimality conditions of Euler-Lagrange type of variational problems in which variational functional depends on the Atangana-Baleanu derivative are proved. Finally, the Hydon method to determine discrete symmetries for a differential equation is employed to construct discrete symmetries for a family of ordinary, partial, and fractional differential equations in [25]. In fractional order systems, it was found that the systems with derivation orders $q_{1}, q_{2}$, and $q_{3}$ generate chaotic behaviors when $q=q_{1}+q_{2}+q_{3}<3$ [13-19]; in other words, chaos continues to exist in these systems for derivation orders less than 3 .

Since the discovery by Strotz et al. [26] of chaos in an economical model, various financial and economical models have been proposed in the literature to better understand this complex dynamic of these systems. Among others, we have the forced Van der Pol model [27], the IS-LM model (Investment Saving-Liquidity Money) [28], the new hyperchaotic finance model [29], and many others [30-33]. In 2001, Ma and Chen [34] proposed a very interesting model to represent the dynamics of financial systems. The analysis of this model revealed interesting dynamics and also an extreme sensitivity to the initial conditions of the variables and parameters of the system. Financial variables such as the exchange rate, gross domestic product, interest rate, and production, to name a few, have a long memory $[35,36]$, i.e., all future fluctuations of these financial variables are influenced by past and present fluctuations. Thus, fractional order derivatives, possessing memory effect, can describe more accurately the dynamics of financial systems than integer order derivatives. In 2008, Chen [14] studied the generalization of the $\mathrm{Ma}$ and Chen system [34], i.e., considered this system with fractional order. Two routes to chaos were found in this fractional order system, namely, the route to chaos via intermittency and period doubling. The lowest derivation order found for which the system generates chaotic behavior was 2.55 in the commensurate fractional-order case and 2.35 in the incommensurate fractional-order case.

Also, to better understand the dynamics of financial systems, a new model was presented by Liao et al. [37] in 2020. Compared to the model proposed by Ma and Chen [34], this new model takes into account the fact that the price index is affected by investment demand. The study of this model was carried out numerically by the authors. It was found that the interaction between three factors in this financial system results in a complex behavior of the system. Complex dynamics behaviors such as period doubling and chaos were found in this system [37]. Chaotic behavior is undesirable in financial systems because it makes predictions in finance and economics impossible and, therefore, constitutes a risk for investments. Then, its control in the presence of uncertainties related to the parameters of the system and external disturbances turned out to be necessary.

Chaos control in financial systems has been widely studied in the literature. Several techniques have been designed for the control of financial chaotic systems [38, 39].
One of these techniques is the sliding mode control. Indeed, as a mathematical model cannot represent a physical situation perfectly, it is necessary to consider the uncertainties linked to the values of the system parameters and also any external disturbances to which a model may be subjected. Therefore, it is better to design a robust controller, i.e., insensitive to uncertainties and external disturbances. The sliding mode control is a powerful technique to robustly control uncertain dynamical systems subject to uncertainties and external disturbances [40-42]. The design of the sliding mode controller has been widely discussed in the literature [43-46]. These different criteria make the sliding mode control the right technique for controlling financial systems with uncertain dynamics. For example, in [38], a fractionalorder sliding mode controller was designed to eliminate the chaotic behavior in an economical system in the presence of model uncertainties and external disturbances.

Motivated by the above discussions, in this paper, chaos in the financial system presented by Liao et al. [37] with fractional order and robust control of this chaotic behavior are investigated. The study of chaos in this system with fractional order is carried out for the commensurate and the incommensurate fractional order to find the minimum effective dimension, i.e., the lowest sum of derivation orders from which chaos arise in the system using analytical methods and numerical simulations. Finally, a sliding mode control law is designed to control the chaos in this fractionalorder financial system with or without uncertainties and external disturbances. Numerical simulations are carried out to show that the controller can suppress chaos in the system and also can stabilize and maintain the system states on the sliding surface.

The remaining part of this paper is structured as follows. In Section 2, some definitions and analytical condition for the existence of chaos in fractional order systems are given. The fractional-order financial system is presented in Section 3. The dynamics study of this fractional-order financial system is carried out in Section 4. In Section 5, a simple but robust fractional-order sliding mode controller is designed to globally and asymptotically stabilize the system. $\mathrm{Nu}-$ merical simulations are performed in Section 6 to verify the analytical results obtained. This work ends with a conclusion in Section 7.

\section{Definitions and Lemma}

Fractional calculus is a generalization of ordinary calculus. Some definitions of fractional derivatives are given in [12]. The most commonly used definitions in the literature are the Grunwald-Letnikov, the Riemann-Liouville, and the Caputo definitions [12].

During this work, we will only use the Caputo fractional derivative because unlike the Riemann-Liouville definition of the fractional derivative which involves initial conditions of the fractional order, this fractional derivative involves initial conditions which take the same form as the case of the integer order, which has a physical interpretation. The Caputo (C) fractional derivative of order $q$ is given by 


$$
{ }_{a}^{C} D_{t}^{q} f(t)=\frac{1}{\Gamma(n-1)} \int_{a}^{t}(t-\tau)^{n-q-1} f^{(n)}(\tau) \mathrm{d} \tau, \quad n-1<q<n,
$$

with $a$ and $t$ which are numbers representing the limits of the operator ${ }_{a}^{C} D_{t}^{q}$. The symbol $\Gamma(\cdot)$ is the gamma function.

Definition 1 (see [47-49]). A saddle equilibrium point is a fixed point at which the equivalent linearized model has at least one eigenvalue in the stable region (eigenvalue with the negative real part) and one in the unstable region (eigenvalue with the positive real part).

Definition 2 (see [47-50]). In a three-dimensional (3D) dynamical system, a saddle fixed point is called a saddle equilibrium point of index 1 if one of its eigenvalues is unstable (positive) and the other two are stable (negative), whereas a saddle fixed point is called a saddle equilibrium point of index 2 if two of its eigenvalues are unstable and the other is stable.

To analyze the stability of a fractional-order system fixed points, the following lemma can be used $[47,48,51]$.

Lemma 1 (see [47]). For an incommensurate fractionalorder system, i.e., a fractional order system in which derivation orders are not the same, an equilibrium point $E^{*}$ of the system is asymptotically stable if the following condition

$$
|\arg (\lambda)|>\frac{\pi}{2 M}
$$

is satisfied for all roots $\lambda$ of the following equation:

$$
\operatorname{det}(\Delta(\lambda))=\operatorname{det}\left(\operatorname{diag}\left(\left[\lambda^{M q_{1}}, \lambda^{M q_{2}}, \ldots, \lambda^{M_{q_{n}}}\right]\right)-J\right)=0,
$$

in which $J$ is the Jacobian matrix of the system evaluated at the equilibrium point $E^{*}$ and $M$ represents the Least Common Multiple (LCM) of the denominators $u_{i}$ 's of $q_{i}$ 's where $q_{i}=$ $\left(v_{i} / u_{i}\right), v_{i}$ and $u_{i} \in Z+$, for $i=1,2, \ldots, n$.

Condition (2) can be rewritten as follows:

$$
\frac{\pi}{2 M}-\min _{i}\left\{\left|\arg \left(\lambda_{i}\right)\right|\right\}<0
$$

So, an equilibrium point $E$ will be asymptotically stable if its roots $\lambda_{i}$ satisfy condition (4).

The term $(\pi / 2 M)-\min _{i}\left\{\left|\arg \left(\lambda_{i}\right)\right|\right\}$ is called the Instability Measure for equilibrium points in Fractional Order Systems (IMFOS). This measure is a necessary [47], but not a sufficient condition for the presence of chaos in a fractional order system [52-54].

\section{The Chaotic Financial System with Fractional Order}

Recently, as reported in the literature [37], the financial model takes into account the interaction between the interest rate $x$, the investment demand $y$, and the price index $z$. The system is described as follows:

$$
\left\{\begin{array}{l}
\frac{\mathrm{d} x}{\mathrm{~d} t}=\mathrm{d} z+(y-e) x, \\
\frac{\mathrm{d} y}{\mathrm{~d} t}=-k y^{2}-l x^{2}+m, \\
\frac{\mathrm{d} z}{\mathrm{~d} t}=-\gamma z-\delta x-\rho y,
\end{array}\right.
$$

in which the parameters $e, k, \gamma, m, l, \rho$, and $\delta$ are constants. The authors numerically investigated the chaotic behaviors in this system. In [37], system (5) exhibits chaotic behavior when $\quad e=0.3, k=0.02, \gamma=1, m=1, l=0.1, \quad \rho=0.05$, $d=1.2, \delta=1$, and initial conditions $(1.2,1.5,1.6)$ are considered (see Figure 1).

In this paper, we consider system (5) with the fractional order. Standard derivatives are replaced by fractional order derivatives as follows:

$$
\left\{\begin{array}{l}
D^{q_{1}} x=\mathrm{d} z+(y-e) x, \\
D^{q_{2}} y=-k y^{2}-l x^{2}+m, \\
D^{q_{3}} z=-\gamma z-\delta x-\rho y,
\end{array}\right.
$$

where $q_{i} \in(0,1)$ and $D^{q_{i}}=\left(\mathrm{d}^{q_{i}} / \mathrm{d} t^{q_{i}}\right)(i=1,2$, and 3$)$. If $q_{1}=q_{2}=q_{3}=q$, then system (6) is called a commensurate fractional-order system; otherwise, it is called an incommensurate order system [47].

The Jacobian matrix of system (6) evaluated at one of its equilibrium points $E^{*}=\left(x^{*}, y^{*}, z^{*}\right)$ is given by

$$
J=\left(\begin{array}{ccc}
y^{*}-e & x^{*} & d \\
-2 l x^{*} & -2 k y^{*} & 0 \\
-\delta & -\rho & -\gamma
\end{array}\right)
$$

When the values of the system parameters are chosen as above, the equilibrium points of system (6) can be calculated by solving the equations $D^{q_{1}} x=0, D^{q_{2}} y=0$, and $D^{q_{3}} z=0$. The system has four equilibrium points which are given by

$$
\begin{aligned}
& E_{1}=(0.049498497,-7.070201517,0.304011579), \\
& E_{2}=(0.076160842,7.069016737,-0.429611679), \\
& E_{3}=(3.087391472,1.529728564,-3.163877901), \\
& E_{4}=(-3.093050811,1.471456216,3.019478000) .
\end{aligned}
$$

The corresponding eigenvalues and their nature are given in Table 1 .

Taking into account Definition 2 and from Table 1, it can be seen that fixed points $E_{1}$ and $E_{2}$ are saddle equilibrium points of index 1 and the others are saddle equilibrium points of index 2 .

Using the Adams-Bashforth-Moulton predictorcorrector method proposed by Diethelm et al. [55], the numerical solution of system (6) can be written as follows: 


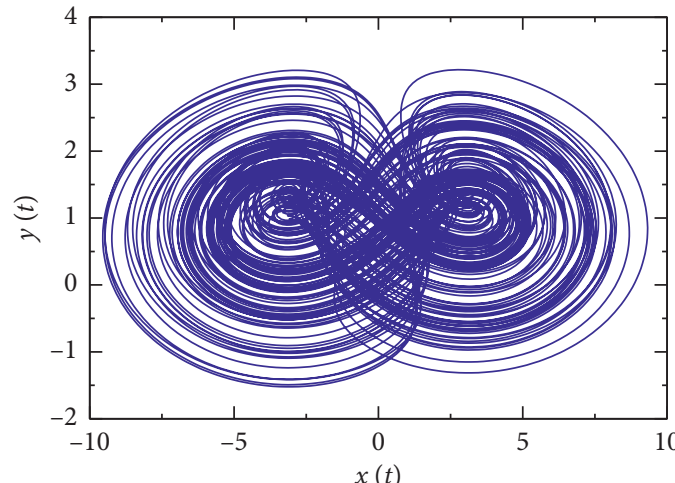

(a)

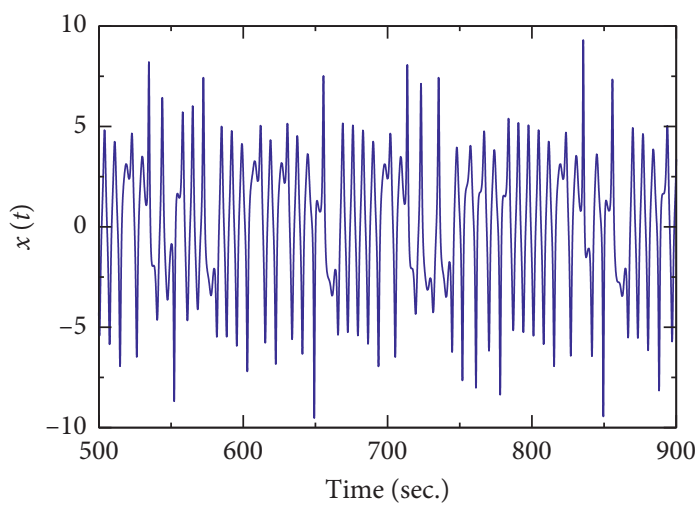

(c)

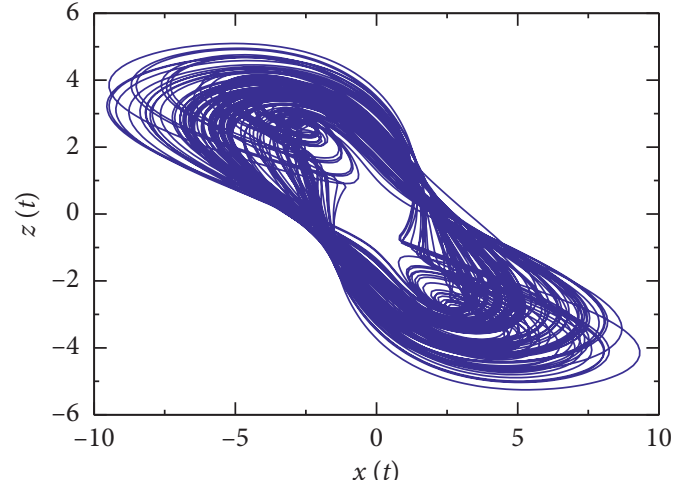

(b)

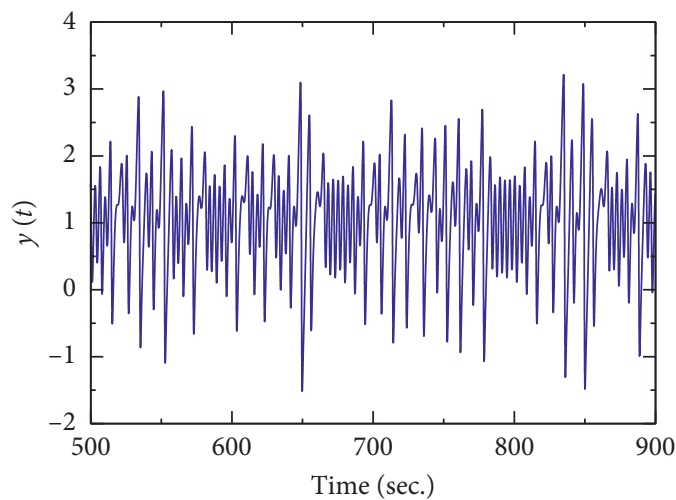

(d)

Figure 1: Phase diagrams and time series of system (5). (a) Projected onto the $x-y$ phase plane. (b) Projected onto the $x-z$ phase plane. (c) Time series of $x$. (d) Time series of $y$.

$$
\left\{\begin{array}{l}
x_{n+1}=x_{0}+\frac{h^{q_{1}}}{\Gamma\left(q_{1}+2\right)}\left[\mathrm{d} z_{n+1}^{p}+\left(y_{n+1}^{p}-e\right) x_{n+1}^{p}\right], \\
\frac{+h^{q_{1}}}{\Gamma\left(q_{1}+2\right)} \sum_{j=0}^{n} \chi_{1, j, n+1}\left(\mathrm{~d} z_{j}+\left(y_{j}-e\right) x_{j}\right), \\
y_{n+1}=y_{0}+\frac{h^{q_{2}}}{\Gamma\left(q_{2}+2\right)}\left[-k\left(y_{n+1}^{p}\right)^{2}-l\left(x_{n+1}^{p}\right)^{2}+m\right], \\
\frac{+h^{q_{2}}}{\Gamma\left(q_{2}+2\right)} \sum_{j=0}^{n} \chi_{2, j, n+1}\left(-k y_{j}^{2}-l x_{j}^{2}+m\right), \\
z_{n+1}=z_{0}+\frac{h^{q_{3}}}{\Gamma\left(q_{3}+2\right)}\left[-\gamma z_{n+1}^{p}-\delta x_{n+1}^{p}-\rho y_{n+1}^{p}\right], \\
\frac{+h^{q_{3}}}{\Gamma\left(q_{3}+2\right)} \sum_{j=0}^{n} \chi_{3, j, n+1}\left(-\gamma z_{j}-\delta x_{j}-\rho y_{j}\right),
\end{array}\right.
$$

where 
TABLE 1: Equilibrium points, corresponding eigenvalues, and their nature.

\begin{tabular}{lcc}
\hline Equilibrium points & Eigenvalues & Nature \\
\hline$E_{1}$ & $\lambda_{1}=-7.1758, \lambda_{2}=-1.1944, \lambda_{3}=0.2828$ & Saddle equilibrium point \\
$E_{2}$ & $\lambda_{1}=6.6418, \lambda_{2}=-0.8428, \lambda_{3}=-0.2828$ & Saddle equilibrium point \\
$E_{3}$ & $\lambda_{1}=-0.7378, \lambda_{2,3}=0.4532 \pm 1.5251 i$ & Saddle equilibrium point \\
$E_{4}$ & $\lambda_{1}=-0.7548, \lambda_{2,3}=0.4337 \pm 1.5487 i$ & Saddle equilibrium point \\
\hline
\end{tabular}

$$
\begin{aligned}
& \left\{\begin{array}{l}
x_{n+1}^{p}=x_{0}+\frac{1}{\Gamma\left(q_{1}\right)} \sum_{j=0}^{n} \theta_{1, j, n+1}\left(\mathrm{~d} z_{j}+\left(y_{j}-e\right) x_{j}\right), \\
y_{n+1}^{p}=y_{0}+\frac{1}{\Gamma\left(q_{2}\right)} \sum_{j=0}^{n} \theta_{2, j, n+1}\left(-k y_{j}^{2}-l x_{j}^{2}+m\right), \\
z_{n+1}^{p}=z_{0}+\frac{1}{\Gamma\left(q_{3}\right)} \sum_{j=0}^{n} \theta_{3, j, n+1}\left(-\gamma z_{j}-\delta x_{j}-\rho y_{j}\right),
\end{array}\right. \\
& \left\{\chi_{i, j, n+1}= \begin{cases}n^{q_{i}+1}-\left(n-q_{i}\right)(n+1)^{q_{i}}, & j=0, \\
(n-j+2)^{q_{i}+1}+(n-j)^{q_{i}+1}-2(n-j+1)^{q_{i}+1}, & 1 \leq j \leq n, \\
1, & j=n+1,\end{cases} \right. \\
& \theta_{i, j, n+1}=\frac{h^{q_{i}}}{q_{i}}\left[(n-j+1)^{q_{i}}-(n-j)^{q_{i}}\right], \quad 1 \leq j \leq n, i=1,2,3 .
\end{aligned}
$$

\section{Dynamics Analysis of the Financial System with Fractional Order}

In this section, the numerical method proposed by Diethelm et al. [55] and presented in the previous section is used to solve numerically system (6) in the commensurate and incommensurate fractional-order cases. The parameters' values defined in Section 3 and initial conditions $\left(x_{0}, y_{0}, z_{0}\right)=(1.2,1.5,1.6)$ will be considered in this part. Using the well-known tools for studying dynamical systems such as phase diagrams, time series, bifurcation diagram, and largest Lyapunov exponent, the dynamics of the financial system with the fractional order will be investigated.

\subsection{Dynamics for Commensurate Fractional-Order System.} Here, we consider system (6) when $q_{1}=q_{2}=q_{3}=q$ (commensurate order). System (6) does not exhibit chaotic behavior if it satisfies the inequality $q<(2 / \pi) \min _{i}\left\{\left|\arg \left(\lambda_{i}\right)\right|\right\}$ [47, 48, 51] with $\lambda_{i}$ being the eigenvalue of the Jacobian matrix of system (6) evaluated at one of its fixed points. For the equilibrium points $E_{3}$ and $E_{4}$, we have $\min _{i}\left\{\left|\arg \left(\lambda_{i}\right)\right|\right\}=1.2819$; so,

$$
q<\frac{2}{\pi} \min _{i}\left\{\left|\arg \left(\lambda_{i}\right)\right|\right\} \approx 0.816
$$

Figure 2 shows that the largest Lyapunov exponent of system (6) with the commensurate fractional order is positive only if $q>0.82$. Therefore, the system does not show chaotic behavior when $q<0.82$. It is found using numerical simulations that the system exhibits a chaotic attractor for $q \geq 0.83$. For $q=0.83$, phase diagrams are shown in Figure 3(a) for the $x-y$ phase plane and in Figure 3(b) for the $x-z$ phase plane. Figures 3(c) and 3(d) show the time series of state variables $x$ and $y$, respectively, for $q=0.83$. As it can be seen, the system exhibits chaotic behavior, and this is confirmed by a positive Lyapunov exponent for $q=0.83$.

Therefore, the minimum effective dimension of system (6) for the commensurate fractional order is $0.83 \times 3=2.49$. Thus, simulation results show that chaos exists in this fractional-order financial system with the derivation order less than 3. For numerical simulations, the step size $h=0.01$ is used.

4.2. Dynamics for Incommensurate Fractional-Order System. When one of the system's derivation orders has a different value from the other two, we get an incommensurate fractional-order system [47].

By observing Figure 4, we can see that the largest Lyapunov exponent of system (6) is positive for $q_{1} \geq 0.57$ with $q_{2}=q_{3}=1$ (see Figure $4(\mathrm{a})$ ), for $q_{2} \geq 0.87$ with $q_{1}=$ $q_{3}=1$ (see Figure 4(b)), and for $q_{3} \geq 0.13$ with $q_{1}=q_{2}=1$ (see Figure $4(\mathrm{c})$ ). For the examples, we consider the following cases: 


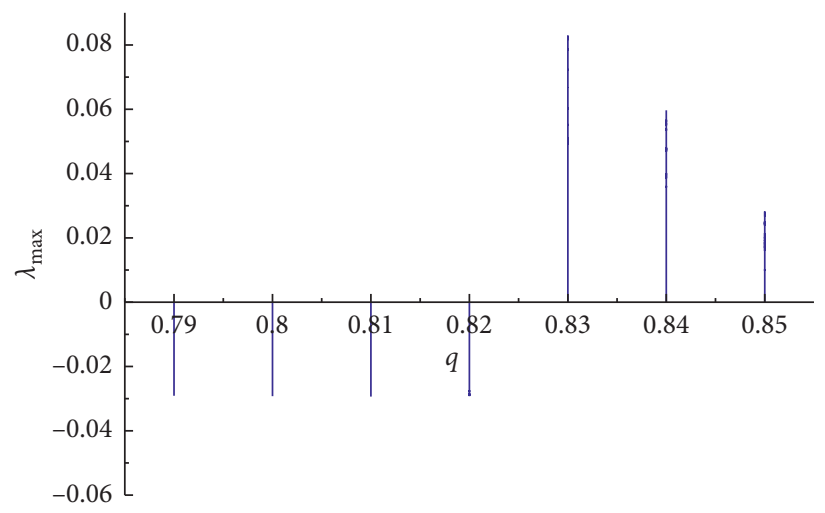

FIgURE 2: The largest Lyapunov exponent with the variation of derivation order $q$.

Case 1: fix $q_{2}=q_{3}=1$, and let us take different values of $q_{1}$. (i) $q_{1}=0.55$ and $q_{2}=q_{3}=1$. In this case, we have $v_{1}=11, u_{1}=20$, and $v_{2}=v_{3}=u_{2}=u_{3}=1, \quad$ so $M=\operatorname{LCM}(20,1,1)=20$ and

$$
\begin{aligned}
\Delta(\lambda)= & \operatorname{diag}\left(\lambda^{M q_{1}}, \lambda^{M q_{2}}, \lambda^{M q_{3}}\right)-J\left(E_{3}\right)=\operatorname{diag}\left(\lambda^{11}, \lambda^{20}, \lambda^{20}\right)-J\left(E_{3}\right), \\
\operatorname{det}(\Delta(\lambda))= & \lambda^{51}-1.22972856 \lambda^{40} \\
& +1.061189142 \lambda^{31}+1.801422624 \lambda^{20} \\
& +0.061189142 \lambda^{11}+1.867529457=0 .
\end{aligned}
$$

By solving equation (13), we have

$$
\begin{aligned}
\text { IMFOS } & =\frac{\pi}{2 M}-\min _{i}\left|\arg \left(\lambda_{i}\right)\right|=\frac{\pi}{40}-0.079176 \\
& =-0.00064<0 .
\end{aligned}
$$

In this case, IMFOS $<0$; therefore, for the derivation orders $q=(0.55,1,1)$, system (6) does not exhibit a chaotic behavior.

(ii) Consider now $q_{1}=0.57$ and $q_{2}=q_{3}=1$; by the same procedure as the above case, we have $M=100$, and

$\operatorname{det}(\Delta(\lambda))=\lambda^{257}-1.22972856 \lambda^{200}+1.061189142 \lambda^{157}+1.801422624 \lambda^{100}+0.061189142 \lambda^{57}+1.867529457=0$

By solving equation (15), we have

$$
\begin{aligned}
\text { IMFOS } & =\frac{\pi}{2 M}-\min _{i}\left|\arg \left(\lambda_{i}\right)\right|=\frac{\pi}{200}-0.015682 \\
& =0.000026>0 .
\end{aligned}
$$

IMFOS > 0; therefore, for the given derivation orders, the system satisfies the necessary condition to present a chaotic attractor. Numerical simulations confirm this conclusion in Figure 5. So, the lowest value for which $q_{1}$ in this case generates a chaotic behavior is 0.57 , where $\lambda_{\max }>0$.

Case 2: fix $q_{1}=q_{3}=1$, and let us take different values of $q_{2}$

(i) Consider $q_{1}=1, q_{2}=0.86$, and $q_{3}=1$; by the same procedure as the above case, we get $M=\operatorname{LCM}(1,50,1)=50$, and

$$
\begin{aligned}
\operatorname{det}(\Delta(\lambda))= & \lambda^{143}+0.061189142 \lambda^{100} \\
& -0.22972856 \lambda^{93}+1.892340326 \lambda^{50} \\
& -0.02972856 \lambda^{43}+1.867529457=0 .
\end{aligned}
$$

By solving equation (17), the IMFOS of the system is

$$
\begin{aligned}
\text { IMFOS } & =\frac{\pi}{2 M}-\min _{i}\left|\arg \left(\lambda_{i}\right)\right| \frac{\pi}{100}-0.027339 \\
& =0.004077>0 .
\end{aligned}
$$

In this case, IMFOS $>0$ but system (6) does not exhibit chaotic behavior $\left(\lambda_{\max }<0\right)$. This shows that the condition IMFOS $\geq 0$ is a necessary condition for chaos to exist and not the sufficient one. 


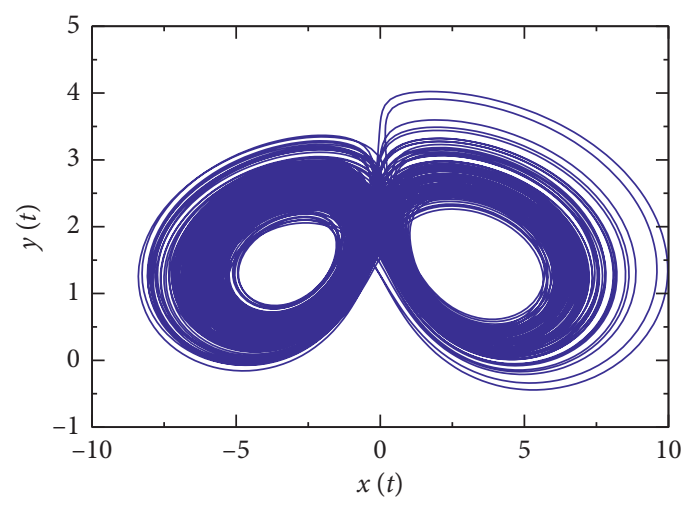

(a)

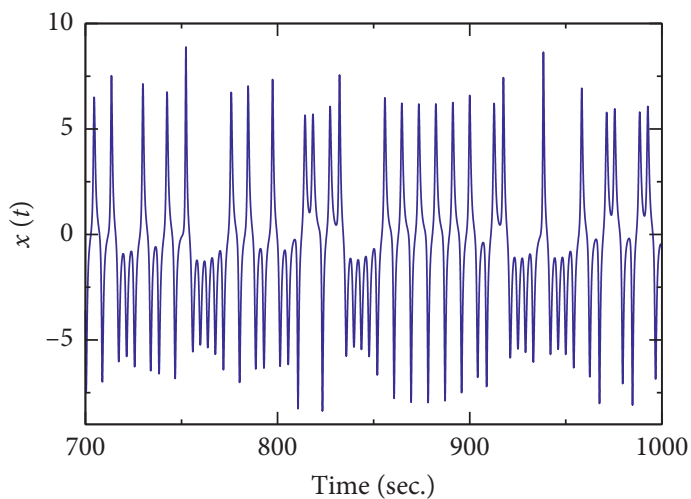

(c)

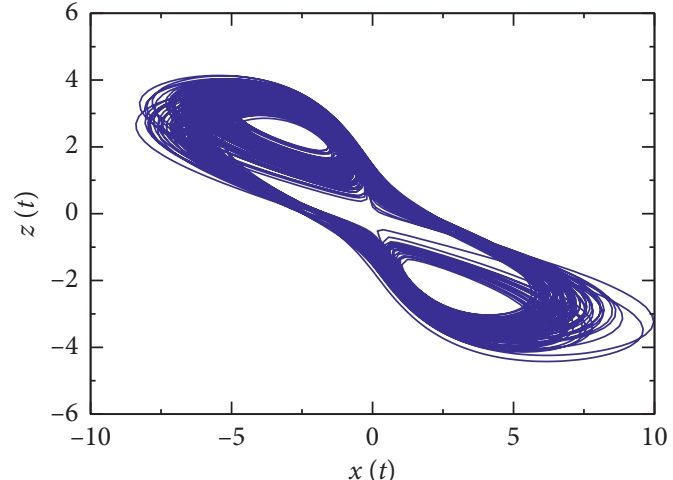

(b)

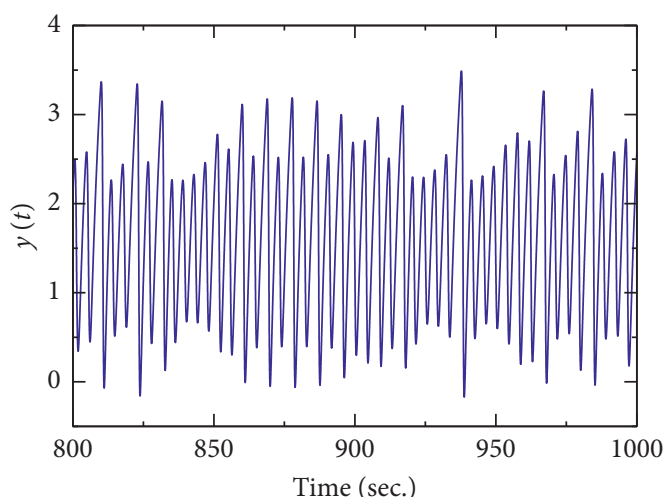

(d)

Figure 3: Phase diagrams and time series of system (6) with fractional order $q=0.83$. (a) Projected onto the $x-y$ phase plane. (b) Projected onto the $x-z$ phase plane. (c) Time series of $x$. (d) Times series of $y$.

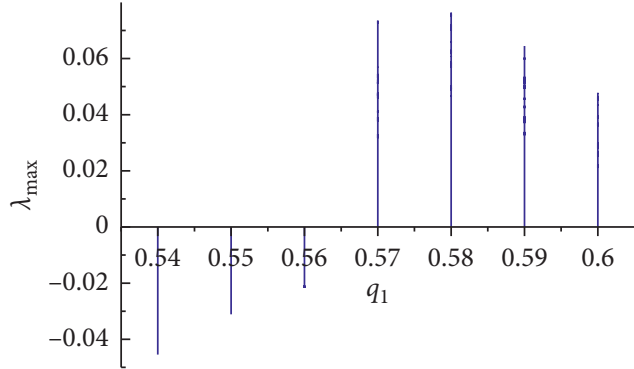

(a)

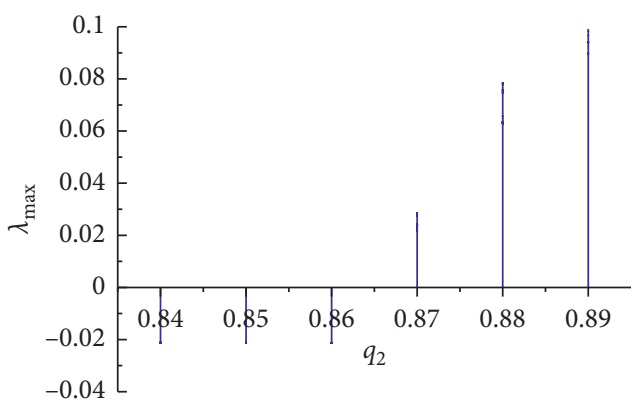

(b)

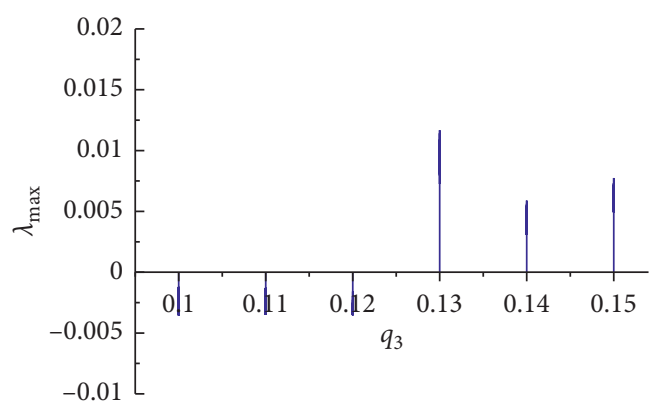

(c)

FIGURE 4: Largest Lyapunov exponents with variation of derivation orders. (a) $q_{1}$, when $q_{2}=q_{3}=1$. (b) $q_{2}$, when $q_{1}=q_{3}=1$. (c) $q_{3}$, when $q_{1}=q_{2}=1$. 


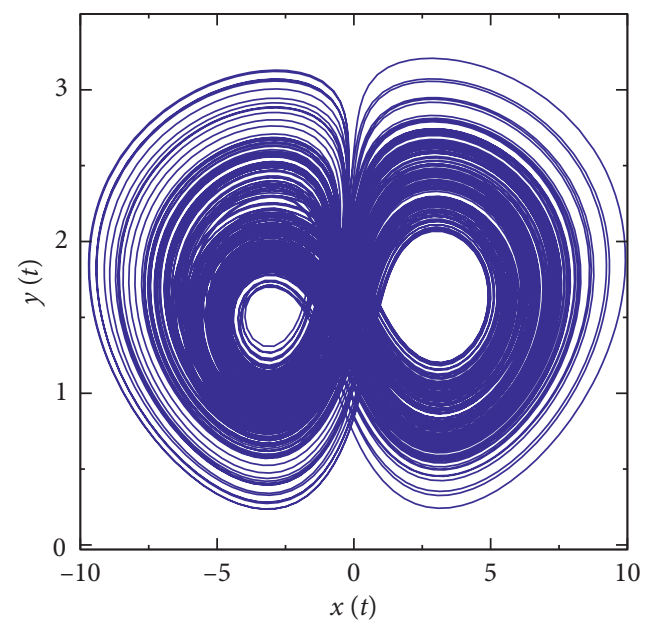

(a)

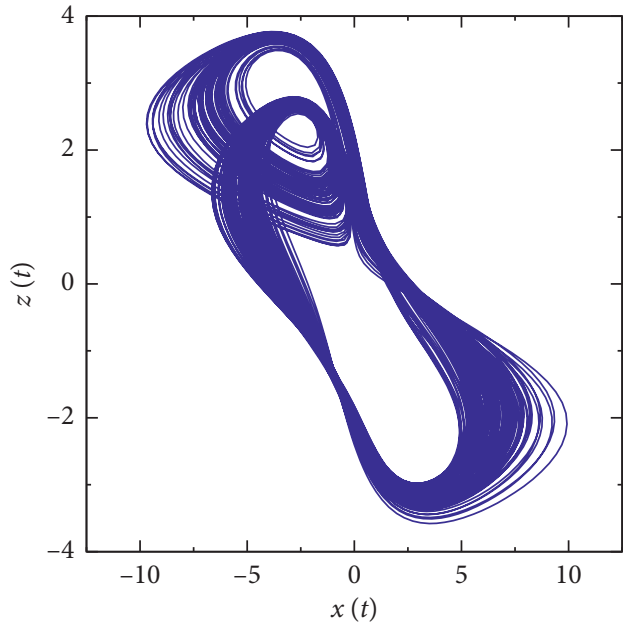

(b)

Figure 5: Phase diagrams of system (6) with fractional orders $q_{1}=0.57$ and $q_{2}=q_{3}=1$ projected onto (a) the $x-y$ phase plane and (b) the $x-z$ phase plane.

(ii) Consider now $q_{1}=1, q_{2}=0.87$, and $q_{3}=1$; by the same procedure as the above case, we have $M=100$, and

$$
\begin{aligned}
& \operatorname{det}(\Delta(\lambda))=\lambda^{287}+0.061189142 \lambda^{200} \\
& -0.22972856 \lambda^{187}+1.892340326 \lambda^{100} \\
& -0.02972856 \lambda^{87}+1.867529457=0 .
\end{aligned}
$$

The system's IMFOS is

$$
\begin{aligned}
\operatorname{IMFOS} & =\frac{\pi}{2 M}-\min _{i}\left|\arg \left(\lambda_{i}\right)\right| \frac{\pi}{200}-0.013605 \\
& =0.002103>0 .
\end{aligned}
$$

In this case, the system exhibits a chaotic behavior as it can be seen in Figure 6, where we can observe the chaotic attractor of the system.

Therefore, the lowest value for which $q_{2}$ in this case generates chaotic behavior is 0.87 , where $\lambda_{\max }>0$.

Case 3: fix $q_{1}=q_{2}=1$, and let us take different values of $q_{3}$.

(i) Consider $q_{1}=1, q_{2}=1$, and $q_{3}=0.10$; then, $M=\operatorname{LCM}(1,1,10)=10$, and

$$
\begin{aligned}
\operatorname{det}(\Delta(\lambda))= & \lambda^{21}+\lambda^{20}-1.168539418 \lambda^{11} \\
& +0.031460582 \lambda^{10}+1.831151184 \lambda \\
& +1.867529457=0 .
\end{aligned}
$$

By solving equation (21), we get

$$
\begin{aligned}
\text { IMFOS } & =\frac{\pi}{2 M}-\min _{i}\left|\arg \left(\lambda_{i}\right)\right| \frac{\pi}{20}-0.135623 \\
& =0.021457>0 .
\end{aligned}
$$

In this case, IMFOS $>0$ but the system does not exhibit chaotic behavior $\left(\lambda_{\max }<0\right)$. (ii) Consider now $q_{1}=1, q_{2}=1$, and $q_{3}=0.13$; then, we have $M=100$, and

$$
\begin{aligned}
\operatorname{det}(\Delta(\lambda))= & \lambda^{213}+\lambda^{200}-1.168539418 \lambda^{113} \\
& +0.031460582 \lambda^{100}+1.831151184 \lambda^{13} \\
& +1.867529457=0 .
\end{aligned}
$$

From equation (23), we get

$$
\begin{aligned}
\text { IMFOS } & =\frac{\pi}{2 M}-\min _{i}\left|\arg \left(\lambda_{i}\right)\right| \frac{\pi}{200}-0.013550 \\
& =0.002158>0 .
\end{aligned}
$$

So, for the derivation orders $q=(1,1,0.13)$, system (6) satisfies the necessary condition for the existence of chaos in the system. In this case, this is confirmed numerically in Figure 7.

Therefore, the lowest value from which $q_{3}$ in this case generates chaotic behavior is $q_{3}=0.13$, where $\lambda_{\max }>0$.

When the value of $q_{3}$ increases from 0.85 to 0.90 , the route to chaos via period doubling is found. The bifurcation diagram and largest Lyapunov exponent when the derivation order $q_{3}$ varies on the closed interval $[0.85,0.90]$ are plotted in Figure 8. Clearly, from the bifurcation diagram, the period-doubling route to chaos can be seen. Figures 9(a)-9(d) show that the system has period-1, period-2, period-4, and chaotic attractors for $q_{3}=0.85,0.87,0.878$, and 0.89 , respectively. Note that the step size used for the numerical simulations in this section is $h=0.01$.

From these three cases, we deduce that the minimum effective dimension of system (6) in the incommensurate fractional-order case is 2.13 .

In the next section, a simple but robust fractional-order sliding mode control law will be designed to control chaos in system (6). 


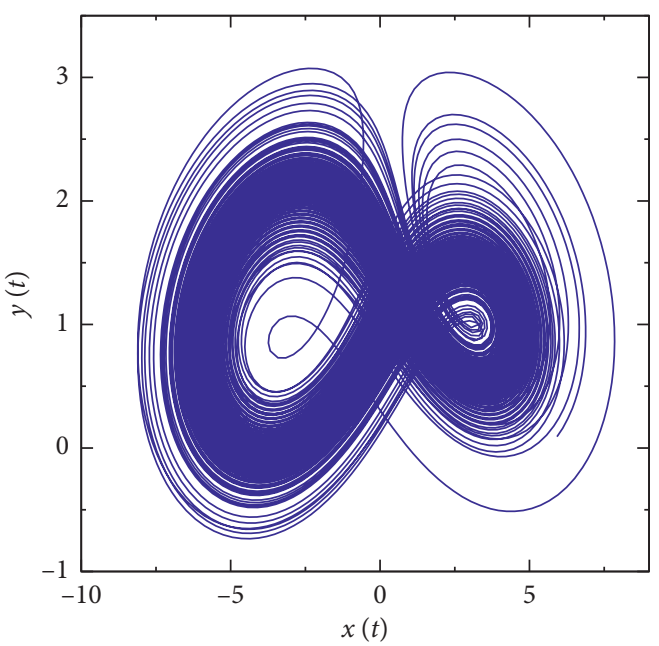

(a)

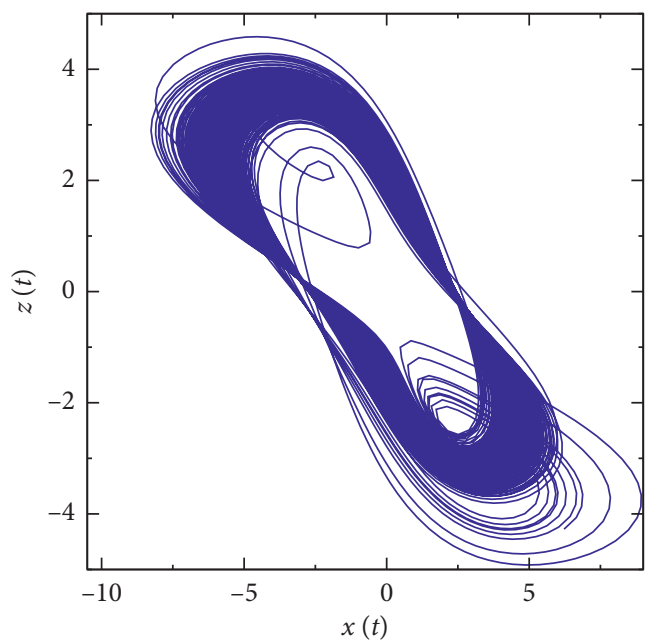

(b)

FIGURE 6: Phase diagrams of system (6) with fractional orders $q_{1}=1, q_{2}=0.87$, and $q_{3}=1$ projected onto (a) the $x-y$ phase plane and (b) the $x-z$ phase plane.

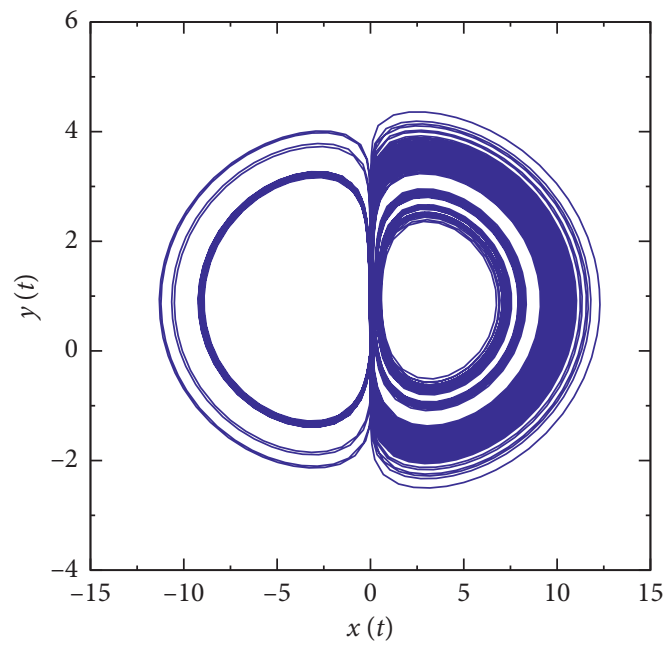

(a)

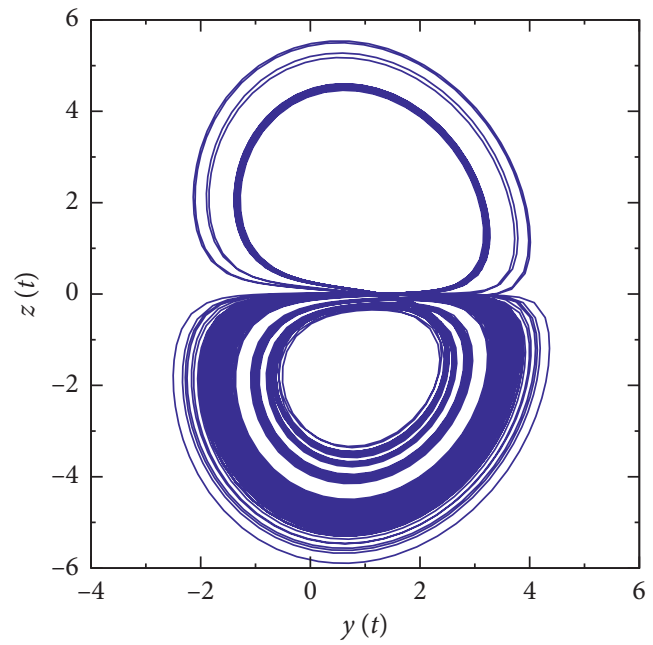

(b)

FIGURE 7: Phase diagrams of system (6) with fractional orders $q_{1}=1, q_{2}=1$, and $q_{3}=0.13$ projected onto (a) the $x-y$ phase plane and (b) the $x-z$ phase plane.

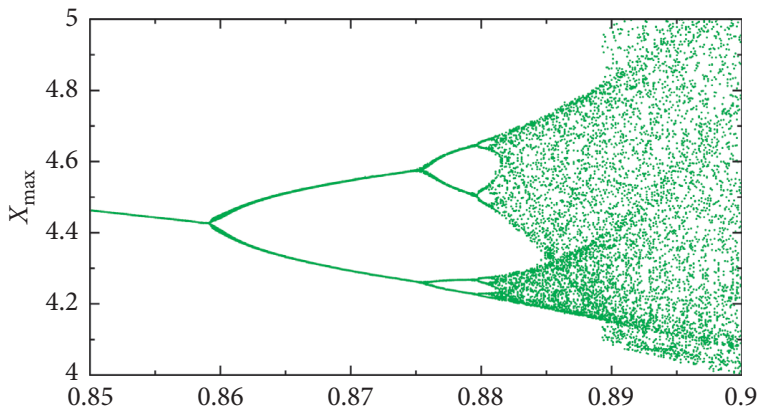

(a)

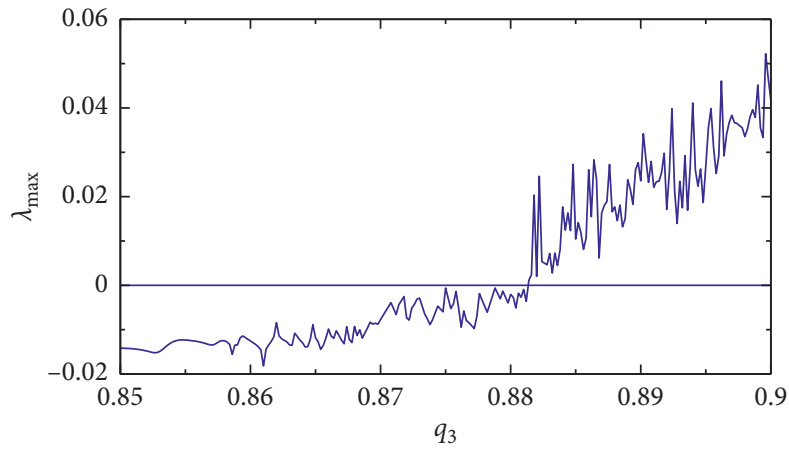

(b)

FiguRE 8: Bifurcation diagram and largest Lyapunov exponent $\left(\lambda_{\max }\right)$ of system (6) with order $q_{3}$. (a) Bifurcation diagram. (b) Largest Lyapunov exponent $\left(\lambda_{\max }\right)$. 


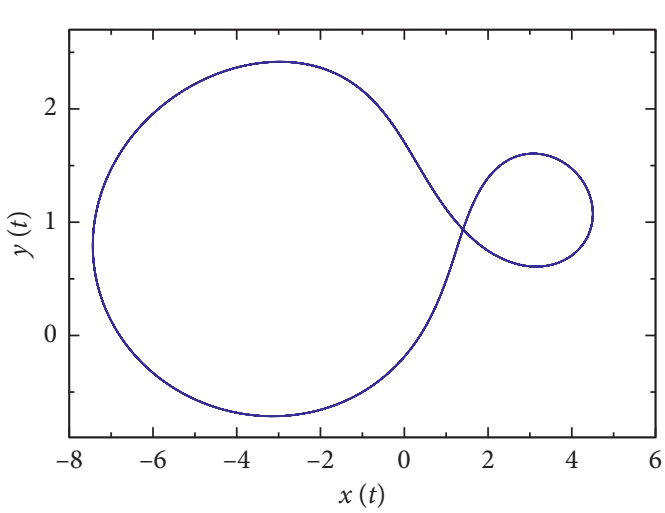

(a)

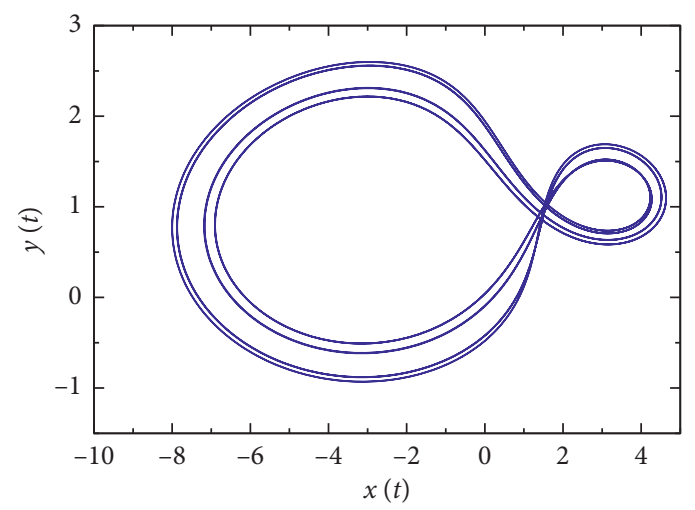

(c)

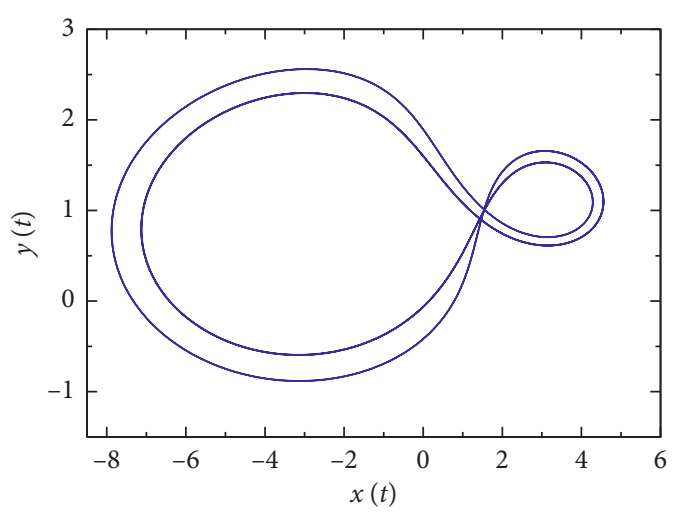

(b)

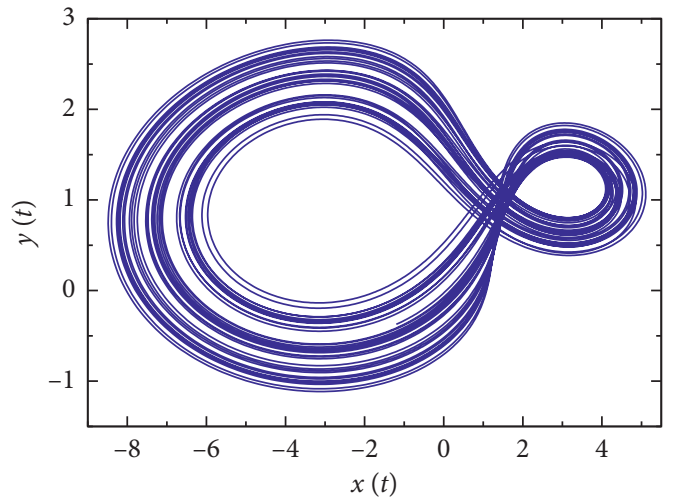

(d)

Figure 9: Phase diagrams showing the period-doubling route to chaos for system (6) with $q_{1}=q_{2}=1$ and fractional order: (a) $q_{3}=0.85$, (b) $q_{3}=0.87$, (c) $q_{3}=0.878$, and (d) $q_{3}=0.89$.

\section{The Sliding Mode Controller Design}

According to the sliding mode control theory, to design a sliding mode controller, we have two steps:

(i) Build a sliding surface, which represents the desired dynamics of the system such as stability

(ii) Develop a control law in a way that the system states are brought towards the sliding surface in a finite time and are maintained in a neighborhood of the sliding surface when time evolves [38]

The sliding mode control law is itself composed of two parts; the first, which is continuous, is called the equivalent control law, and the second is discontinuous. The equivalent control law describes the behavior of the system to be controlled when its trajectories are on the sliding surface. The discontinuous reaching law ensures the convergence of all the system states towards the sliding surface.

To control chaos in fractional-order financial system (6), the controller $u(t)$ is added at the level of the second state equation as follows:

$$
\left\{\begin{array}{l}
D^{q_{1}} x=\mathrm{d} z+(y-e) x, \\
D^{q_{2}} y=-k y^{2}-l x^{2}+m+u, \\
D^{q_{3}} z=-\gamma z-\delta x-\rho y .
\end{array}\right.
$$

Motivated by literature $[38,43]$, as a choice for the sliding surface, we choose the following fractional-order sliding surface:

$$
\begin{aligned}
\sigma(t) & =D^{q_{2}-1} y(t)+D^{-1}\left(k y^{2}(t)+l x^{2}(t)+n y(t)\right) \\
& =D^{q_{2}-1} y(t)+\int_{0}^{t}\left(k y^{2}(\tau)+l x^{2}(\tau)+\eta y(\tau)\right) \mathrm{d} \tau,
\end{aligned}
$$

where $\eta$ is an arbitrary positive constant. In the sliding mode, the invariance conditions of the surface must be satisfied and are defined as follows (i.e., the sliding surface and its derivative must satisfy the relations which appear in the following equation):

$$
\begin{aligned}
\sigma(t) & =0, \\
\frac{\mathrm{d}}{\mathrm{d} t} \sigma(t) & =\sigma^{\prime}(t)=0 .
\end{aligned}
$$

From equations (26) and (27), we get

$$
D^{q_{2}} y(t)=-\left(k y^{2}(t)+l x^{2}(t)\right)+\eta y(t)
$$

From system (25) and equation (28), we obtain the equivalent control law as follows: 


$$
\begin{aligned}
u_{\mathrm{eq}} & =\frac{\mathrm{d}^{q_{2}} y}{\mathrm{~d} t^{q_{2}}}+k y^{2}+l x^{2}-m \\
& =-\left(k y^{2}+l x^{2}+\eta y\right)+k y^{2}+l x^{2}-m \\
& =-\eta y-m .
\end{aligned}
$$

Regarding the discontinuous reaching law, it is chosen as follows:

$$
u_{r}=G_{r} \operatorname{sign}(\sigma),
$$

in which

$$
\operatorname{sign}(\sigma)= \begin{cases}+1, & \text { if } \sigma>0 \\ 0, & \text { if } \sigma=0 \\ -1, & \text { if } \sigma<0\end{cases}
$$

and $G_{r}$ is the gain of the controller. Finally, the total control law has the following form:

$$
u(t)=u_{\mathrm{eq}}(t)+u_{r}(t)=-\eta y-m+G_{r} \operatorname{sign}(\sigma) .
$$

Theorem 1. System (6) with control law (32), i.e., system (25) is globally and asymptotically stable if the controller gain $G_{r}<0$.

Proof. For this, let us choose the Lyapunov quadratic function as follows:

$$
V=\frac{1}{2} \sigma^{2},
$$

and its derivative gives

$$
\begin{aligned}
\dot{V} & =\sigma \dot{\sigma}=\sigma\left[D^{q_{2}} y+k y^{2}+l x^{2}+\eta y\right] \\
& =\sigma\left[-k y^{2}-l x^{2}+m+u+k y^{2}+l x^{2}+\eta y\right] \\
& =\sigma\left[-k y^{2}-l x^{2}+m-n y-m+G_{r} \operatorname{sign}(\sigma)+k y^{2}+l x^{2}+\eta y\right] \\
& =\sigma\left[G_{r} \operatorname{sign}(\sigma)\right] \\
& =G_{r}|\sigma|<0 .
\end{aligned}
$$

Therefore, we have found a Lyapunov function which satisfies the conditions of Lyapunov theorem, i.e., $V>0$ and $V^{\prime}<0$. Thus, system (25) with sliding mode control law (32) is globally and asymptotically stable.

Theorem 2. Suppose that system (25) is perturbed by uncertainties and an external disturbance. Thus, the system has the following form:

$$
\left\{\begin{array}{l}
D^{q_{1}} x=\mathrm{d} z+(y-e) x, \\
D^{q_{2}} y=-k y^{2}-l x^{2}+m+\Delta g(x, y, z)+p(t)+u, \\
D^{q_{3}} z=-\gamma z-\delta x-\rho y,
\end{array}\right.
$$

in which $\Delta g(x, y, z)$ and $p(t)$ are supposed to be bounded, i.e., $|\Delta g(x, y, z)| \leq \mu_{1}$ and $|(t)| \leq \mu_{2}$, where $\mu_{1}$ and $\mu_{2}$ are positive constants. System (35) with sliding mode control law (32) is globally and asymptotically stable if $G_{r}<-\left(\mu_{1}+\mu_{2}\right)$.

Proof. For this, let us choose Lyapunov quadratic function (33); thus, we have

$$
\begin{aligned}
\dot{V}= & \sigma \dot{\sigma}=\sigma\left[D^{q_{2}} y+k y^{2}+l x^{2}+\eta y\right] \\
= & \sigma\left[-k y^{2}-l x^{2}+m+\Delta g(x, y, z)\right. \\
& \left.+p(t)+u+k y^{2}+l x^{2}+\eta y\right] \\
= & \sigma\left[\Delta g(x, y, z)+p(t)+G_{r} \operatorname{sign}(\sigma)\right] \\
& \leq\left(G_{r}+\mu_{1}+\mu_{1}\right)|\sigma|<0 .
\end{aligned}
$$

Thus, the proof is achieved.

\section{Numerical Simulations}

This part of the paper presents three illustrative examples to verify the effectiveness of the proposed control technique. Numerical simulations are carried out with initial conditions $\left(x_{0}, y_{0}, z_{0}\right)=(1.2,1.5,1.6), \eta=0.5$, and $G_{r}=-2$. For the commensurate fractional-order case, $q_{1}=q_{2}=q_{3}=q=0.83$ is chosen and for which the system is chaotic (see Figure 3 ). For the incommensurate fractionalorder case, $q_{1}=1, q_{2}=0.88$, and $q_{3}=1$ are chosen. For this choice, the largest Lyapunov exponent is positive (see Figure 4(b)). The corresponding phase diagrams and time series are shown in Figure 10. Note that the controller can be activated in the system at any time. In this part, it is activated at $t=20 \mathrm{sec}$.

Case 1: commensurate order without uncertainty and an external disturbance.

As it was shown above, system (25) without the controller $u(t)$ is chaotic for $q_{1}=q_{2}=q_{3}=q=0.83$. Now, applying controller (32) to the system, simulation results can be seen in Figure 11. From this figure, it is observed that control law (32) can effectively asymptotically stabilize the state variables of system (25) (see Figures 11(a)-11(c)). Besides, the time series of the sliding surface $\sigma(t)$ is plotted in Figure 11(d). From this figure, it can be observed that the controller stabilizes the trajectories of the system on the sliding surface and maintains them on this surface when time evolves.

Case 2: commensurate order with uncertainty and an external disturbance.

In this part, we disturb the fractional-order financial system by an uncertainty defined by $\Delta g(x$, $y, z)=0.2 \sin \left(\sqrt{x^{2}+y^{2}+z^{2}}\right)$ and an external disturbance $p(t)=0.5 \sin (2 t)$, where $|\Delta g(x, y, z)| \leq \mu_{1}=0.2$ and $|p(t)| \leq \mu_{2}=0.5$. The time series of system (25) state variables (see Figures 12(a)-12(c)) and the time series of sliding surface (26) (see Figure 12(d)) in the presence of control law (32) can be seen through Figure 12. From this figure, we can see that the controller stabilizes the system in the presence of uncertainty and external disturbance. 


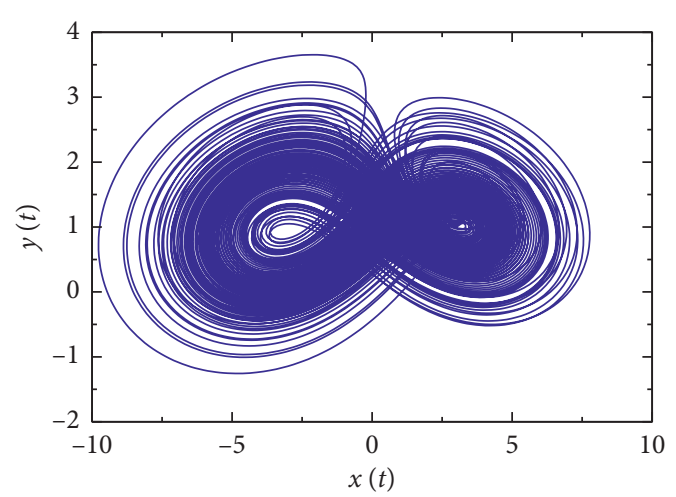

(a)

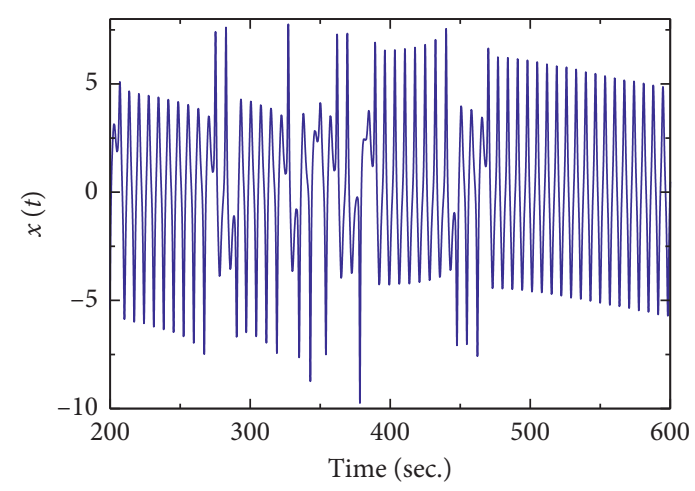

(c)

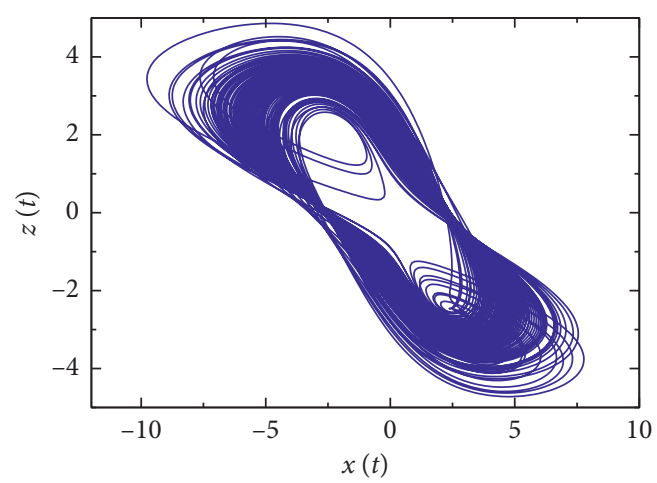

(b)

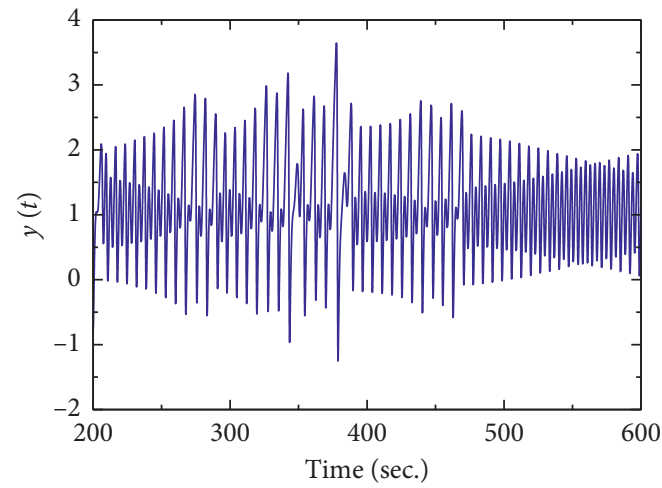

(d)

Figure 10: Phase diagrams and time series of system (6) with $q_{1}=1, q_{2}=0.88$, and $q_{3}=1$. (a) Projected onto the $x-y$ phase plane. (b) Projected onto the $x-z$ phase plane. (c) Time series of $x$. (d) Time series of $y$.

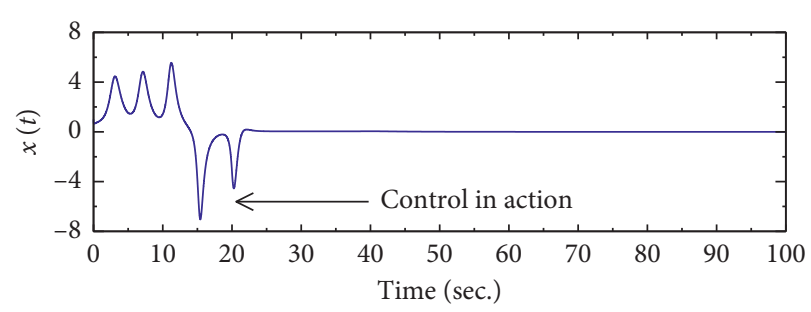

(a)

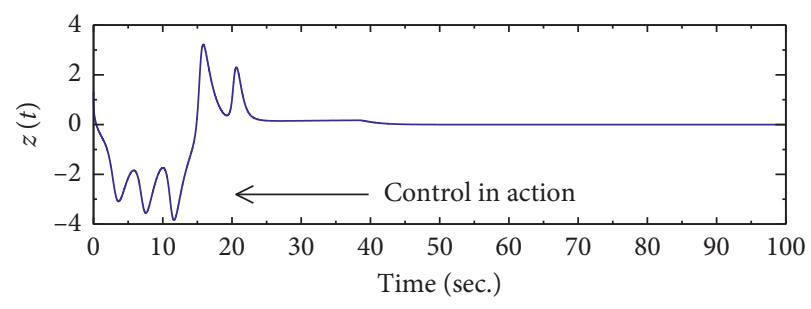

(c)

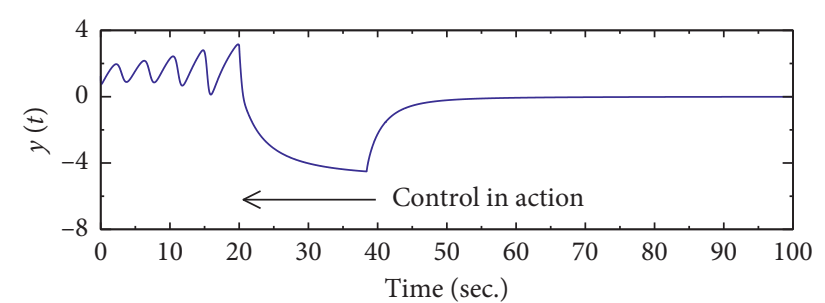

(b)

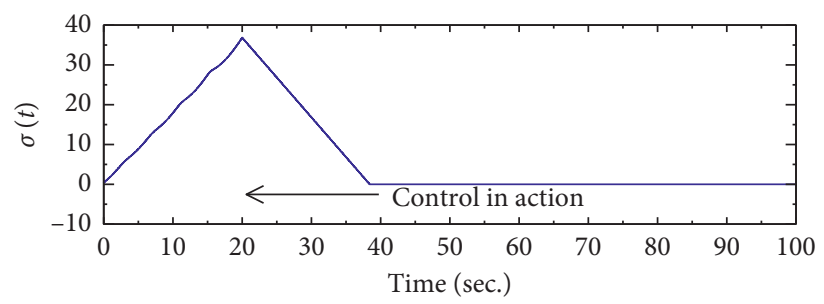

(d)

FIGURE 11: The time series of the controlled commensurate fractional-order system state variables and corresponding time series of the sliding surface without uncertainty and external disturbance (the control input is activated at $t=20 \mathrm{sec}$ ). 


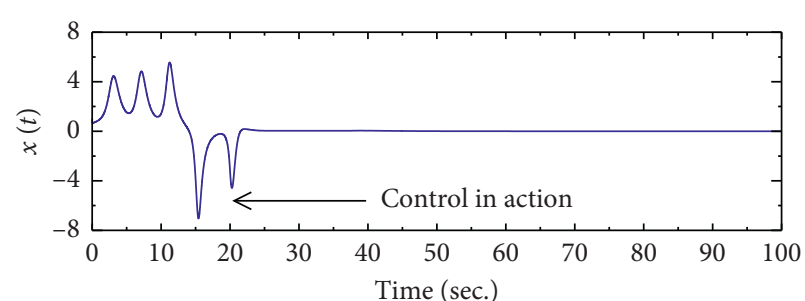

(a)

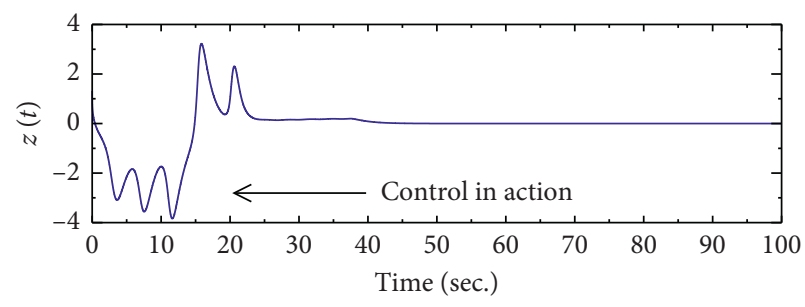

(c)

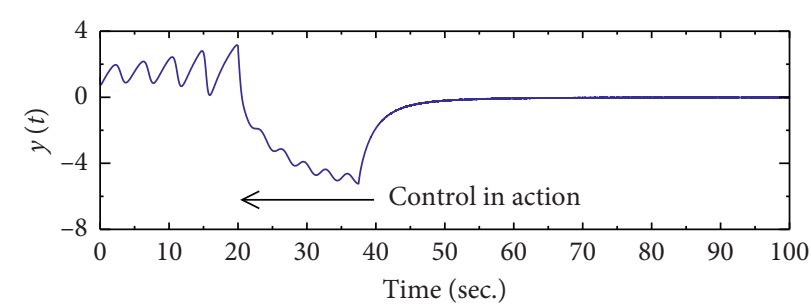

(b)

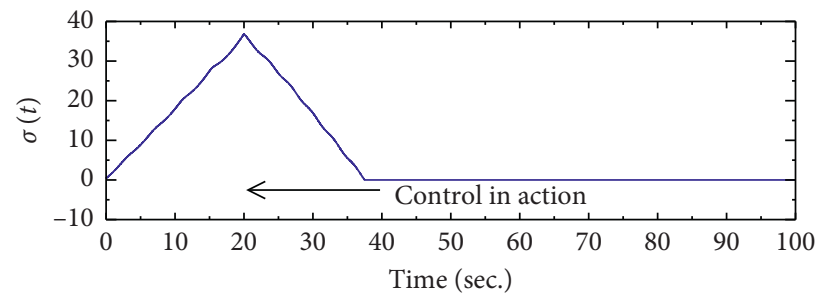

(d)

FIGURE 12: The time series of the controlled commensurate fractional-order system state variables and corresponding time series of the sliding surface with uncertainty and external disturbance (the control input is activated at $t=20 \mathrm{sec}$ ).

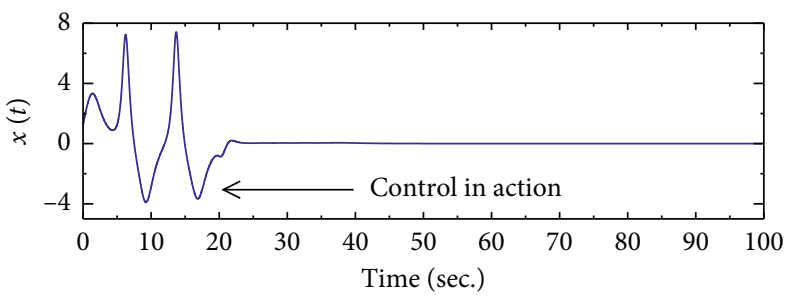

(a)

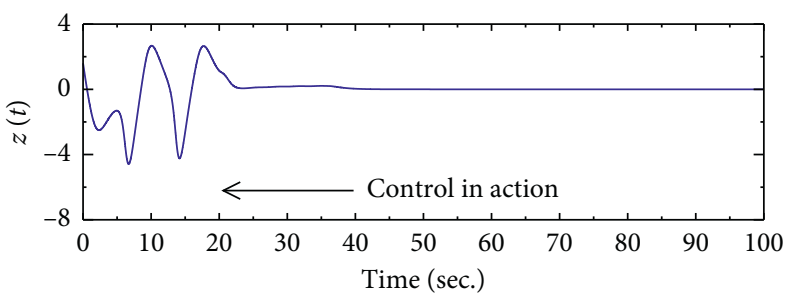

(c)

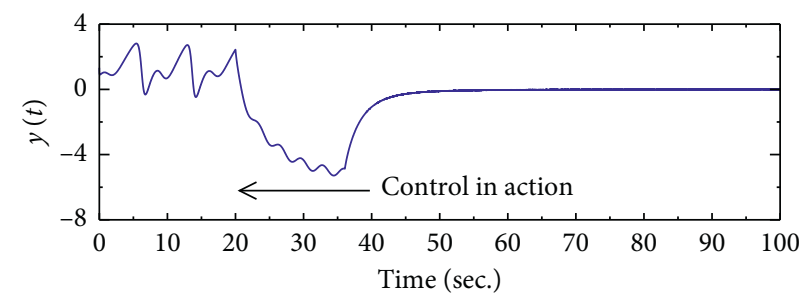

(b)

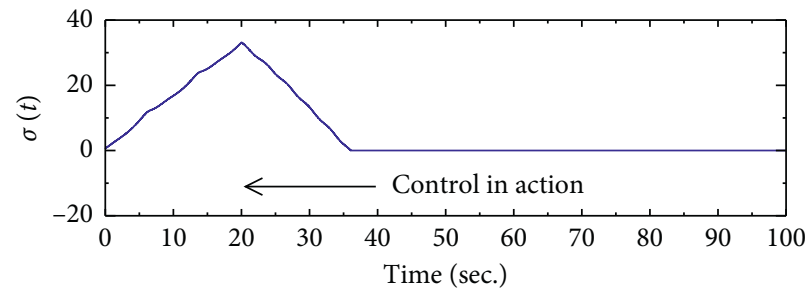

(d)

FIGURE 13: The time series of the controlled incommensurate fractional-order system state variables and corresponding time series of the sliding surface with uncertainty and external disturbance (the control input is activated at $t=20 \mathrm{sec}$ ).

Case 3: incommensurate order with uncertainty and an external disturbance.

In this case, we disturb financial system (25) with the incommensurate fractional order by the same uncertainty and external disturbance as case 2 .

The time series of system (25) state variables and the time series of the sliding surface in the presence of control law (32) are shown in Figure 13. From this figure, it can be observed that the state variables of the system are stabilized.

In each figure, the time series of the sliding surface is plotted to show the ability of the controller to bring back all the system states onto the sliding surface and to maintain them on this surface when time evolves. Simulation results show that controller (32) is able to stabilize systems (25) and (35) in commensurate and incommensurate fractional-order cases. Therefore, the designed control law can suppress chaos in this fractional-order financial system in the presence or not of uncertainty and external disturbance.

\section{Conclusions}

In this paper, the dynamics of a financial system with the fractional order as well as the robust chaos control in this system are studied analytically, and numerical simulations are performed to confirm the analytical results. The existence of chaos in this study is validated by a positive Lyapunov exponent and by an analytical condition existing in the 
literature. The fractional order system exhibits rich dynamics behaviors such as periodic and chaotic behaviors. A perioddoubling route to chaos is found in this system. Numerical simulations revealed that chaos exists in this fractional order system for derivation orders less than 3 . The lowest derivation order found to have chaos in the commensurate fractional-order case is 2.49 and 2.13 for the incommensurate fractional-order case. Regarding the robust control of chaos in the system, by using Lyapunov's stability theorem, a simple but robust fractional-order sliding mode control law has been designed to stabilize the chaotic trajectories of the fractional-order financial system in the presence or not of uncertainty and external disturbance. It should be noted that the controller has been applied only at the investment demand state equation to fully control the system. Numerical simulations show that this controller is effective and can control the financial system with commensurate and incommensurate fractional orders. In [14], Chen studied the fractional order version of the financial system proposed by $\mathrm{Ma}$ and Chen [34]. The lowest derivation order obtained in [14] for chaos to exist is 2.55 in the commensurate order case and 2.35 in the incommensurate order case. Compared to the fractional order version of Liao et al. [37] proposed in this paper, the emergence of chaos is enhanced in both cases. It is well known that time delay can affect the behavior of dynamical systems. For future works, chaotic dynamics analysis of this fractional-order financial system with time delay can be considered.

\section{Data Availability}

No data were used to support the findings of this study.

\section{Conflicts of Interest}

The authors declare that they have no conflicts of interest.

\section{Acknowledgments}

The authors thank IMSP-UAC and German Academic Exchange Service (DAAD) for financial support under the programme "In-Country/In-Region Scholarship Programme" (Funding program no. 57506853).

\section{References}

[1] W. L. Ditto, "Applications of chaos in biology and medicine," AIP Conference Proceedings, vol. 376, no. 1, pp. 175-201, 1996.

[2] J. Ma, C. N. Wang, J. Tang, and Y. F. Xia, "Suppression of the spiral wave and turbulence in the excitability-modulated media," International Journal of Theoretical Physics, vol. 48, no. 1, pp. 150-157, 2009.

[3] Y. Liu and Q. Yang, "Dynamics of a new Lorenz-like chaotic system," Nonlinear Analysis: Real World Applications, vol. 11, no. 4, pp. 2563-2572, 2010.

[4] E. N. Lorenz, "Deterministic nonperiodic flow," Journal of the Atmospheric Sciences, vol. 20, no. 2, pp. 130-141, 1963.

[5] G. Chen and T. Ueta, "Yet another chaotic attractor," International Journal of Bifurcation and Chaos, vol. 9, no. 7, pp. 1465-1466, 1999.
[6] J. Lü, G. Chen, and S. Zhang, "Dynamical analysis of a new chaotic attractor," International Journal of Bifurcation and Chaos, vol. 12, no. 5, pp. 1001-1015, 2002.

[7] R. B. Leipnik and T. A. Newton, "Double strange attractors in rigid body motion with linear feedback control," Physics Letters A, vol. 86, no. 2, pp. 63-67, 1981.

[8] S. Dadras, H. R. Momeni, and V. J. Majd, "Sliding mode control for uncertain new chaotic dynamical system," Chaos, Solitons \& Fractals, vol. 41, no. 4, pp. 1857-1862, 2009.

[9] M. Roopaei, B. R. Sahraei, and T. C. Lin, "Adaptive sliding mode control in a novel class of chaotic systems," Communications in Nonlinear Science and Numerical Simulation, vol. 15, no. 12, pp. 4158-4170, 2010.

[10] M. T. Yassen, "Chaos control of chaotic dynamical systems using backstepping design," Chaos, Solitons \& Fractals, vol. 27, no. 2, pp. 537-548, 2006.

[11] Q. Jia, "Chaos control and synchronization of the Newtonleipnik chaotic system," Chaos, Solitons \& Fractals, vol. 35, no. 4, pp. 814-824, 2008.

[12] I. Podlubny, Fractional Differential Equations, Academic Press, Cambridge, MA, USA, 1999.

[13] C. Li and G. Peng, "Chaos in Chen's system with a fractional order," Chaos, Solitons \& Fractals, vol. 22, no. 2, pp. 443-450, 2004.

[14] W. C. Chen, "Nonlinear dynamics and chaos in a fractionalorder financial system," Chaos, Solitons \& Fractals, vol. 36, no. 5, pp. 1305-1314, 2008.

[15] V. Daftardar-Gejji and S. Bhalekar, "Chaos in fractional ordered Liu system," Computers \& Mathematics with Applications, vol. 59, no. 3, pp. 1117-1127, 2010.

[16] X. Y. Wang, Y. J. He, and M. J. Wang, "Chaos control of a fractional order modified coupled dynamos system," Nonlinear Analysis: Theory, Methods \& Applications, vol. 71, no. 12, pp. 6126-6134, 2009.

[17] T. T. Harley, C. F. Lorenzo, and H. K. Qammar, "Chaos in a fractional order Chua's system," IEEE Transactions on Circuits and Systems I, vol. 42, no. 8, pp. 485-490, 1995.

[18] I. Grigorenko and E. Grigorenko, "Chaotic dynamics of the fractional Lorenz system," Physical Review Letters, vol. 91, no. 3, Article ID 034101, 2003.

[19] W. M. Ahmad and J. C. Sprott, "Chaos in fractional-order autonomous nonlinear systems," Chaos, Solitons \& Fractals, vol. 16, no. 2, pp. 339-351, 2003.

[20] I. Petrás, Fractional-Order Nonlinear Systems: Modeling, Analysis and Simulation, Springer Science and Bussiness Media, Berlin, Germany, 2011.

[21] C. Li and W. Deng, "Remarks on fractional derivatives," Applied Mathematics and Computation, vol. 187, no. 2, pp. 777-784, 2007.

[22] Y. Chatibi, A. Ouhadan, E. Hassan El Kinani, and A. Ouhadan, "Lie symmetry analysis of conformable differential equations," AIMS Mathematics, vol. 4, no. 4, pp. 1133-1144, 2019.

[23] Y. Chatibi, E. H. El kinani, and A. Ouhadan, "Lie symmetry analysis and conservation laws for the time fractional blackscholes equation," International Journal of Geometric Methods in Modern Physics, vol. 17, no. 1, p. 14, 2020.

[24] Y. Chatibi, E. H. El Kinani, and A. Ouhadan, "Variational calculus involving nonlocal fractional derivative with mittagleffler kernel," Chaos, Solitons \& Fractals, vol. 118, pp. 117121, 2019.

[25] Y. Chatibi, E. H. El kinani, and A. Ouhadan, "On the discrete symmetry analysis of some classical and fractional differential 
equations," Mathematical Methods in the Applied Sciences, vol. 44, no. 4, pp. 2868-2878, 2019.

[26] R. H. Strotz, J. C. McAnulty, and J. B. Naines, "Goodwin's nonlinear theory of the business cycle: an electro-analog solution," Econometrica, vol. 21, no. 3, pp. 390-411, 1953.

[27] A. C. L. Chian, "Nonlinear dynamics and chaos in macroeconomics," International Journal of Theoretical and Applied Finance, vol. 3, no. 3, p. 601, 2000.

[28] L. De Cesare and M. Sportelli, "A dynamic IS-LM model with delayed taxation revenues," Chaos, Solitons \& Fractals, vol. 25, no. 1, pp. 233-244, 2005.

[29] H. Yu, G. Cai, and Y. Li, "Dynamic analysis and control of a new hyperchaotic finance system," Nonlinear Dynamics, vol. 67 , no. 3, pp. 2171-2182, 2012.

[30] L. Chen and G. Chen, "Controlling chaos in an economic model," Physica A: Statistical Mechanics and Its Applications, vol. 374, no. 1, pp. 349-358, 2007.

[31] W. Wu, Z. Chen, and W. H. Ip, "Complex nonlinear dynamics and controlling chaos in a cournot duopoly economic model," Nonlinear Analysis: Real World Applications, vol. 11, no. 5, pp. 4363-4377, 2010.

[32] S. Invernizzi and A. Medio, "On lags and chaos in economic dynamic models," Journal of Mathematical Economics, vol. 20, no. 6, pp. 521-550, 1991.

[33] D. A. Hsieh, "Chaos and nonlinear dynamic: application to financial markets," The Journal of Finance, vol. 46, no. 5, pp. 1839-1877, 1991.

[34] J. H. Ma and Y. S. Chen, "Study for the bifurcation topological structure and the global complicated character of a kind of nonlinear finance system, I," Applied Mathematics and Mechanics, vol. 22, no. 11, pp. 1240-1251, 2001.

[35] E. Panas, "Long memory and chaotic models of prices on the London metal exchange," Resources Policy, vol. 27, no. 4, pp. 235-246, 2001.

[36] B. J. West and S. Picozzi, "Fractional Langevin model of memory in financial time series," Physical Review E, Statistical, Nonlinear, and Soft Matter Physics, vol. 65, no. 3, Article ID 037106, 2002.

[37] Y. Liao, Y. Zhou, F. Xu, and X. B. Shu, "A study on the complexity of a new chaotic financial system," Complexity, vol. 2020, Article ID 8821156, 5 pages, 2020.

[38] S. Dadras and H. R. Momeni, "Control of a fractional-order economical system via sliding mode," Physica A: Statistical Mechanics and Its Applications, vol. 389, no. 12, pp. 24342442, 2010.

[39] M. S. Abd-Elouahab, N. E. Hamri, and J. Wang, "Chaos control of a fractional order financial system," Mathematical Problems in Engineering, vol. 2010, Article ID 270646, 18 pages, 2010.

[40] W. Perruquetti and J. P. Barbot, Sliding Mode Control in Engineering, Marcel Dekker Inc., New York, NY, USA, 2002.

[41] J. E. Slotine and W. Li, Applied Nonlinear Control, Prentice Hall, Englewood Cliffs, NJ, USA, 1991.

[42] H. K. Khalil, Nonlinear Systems, Prentice Hall, Englewood Cliffs, NJ, USA, 1996.

[43] M. R. Faieghi, H. Delavari, and D. Baleanu, "Control of an uncertain fractional-order Liu system via fuzzy fractionalorder sliding mode control," Journal of Vibration and Control, vol. 18, no. 9, pp. 1366-1374, 2012.

[44] D. Y. Chen, Y. X. Liu, X. Y. Ma, and R. F. Zhang, "Control of a class of fractional-order chaotic systems via sliding mode," Nonlinear Dynamics, vol. 67, no. 1, pp. 893-901, 2012.

[45] C. Yin, S. M. Zhong, and W. F. Chen, "Design of sliding mode controller for a class of fractional-order chaotic systems,"
Communications in Nonlinear Science and Numerical Simulation, vol. 17, no. 1, pp. 356-366, 2012.

[46] A. J. Muñoz-Vázquez, V. Parra-Vega, and A. Sánchez-Orta, "Fractional integral sliding modes for robust tracking of nonlinear systems," Nonlinear Dynamics, vol. 87, no. 2, pp. 895-901, 2017.

[47] M. S. Tavazoei and M. Haeri, "Chaotic attractors in incommensurate fractional order systems," Physica D: Nonlinear Phenomena, vol. 237, no. 20, pp. 2628-2637, 2008.

[48] M. S. Tavazoei and M. Haeri, "A necessary condition for double scroll attractor existence in fractional order systems," Physics Letters A, vol. 367, no. 1-2, pp. 102-113, 2007.

[49] E. Campos-Cantón, J. G. Barajas-Ramirez, G. Solis-Perales, and R. Femat, "Multiscroll attractors by switching systems," Chaos: An Interdisciplinary Journal of Nonlinear Science, vol. 20, no. 1, Article ID 013116, 2010.

[50] L. Chua, M. Komuro, and T. Matsumoto, "The double scroll family," IEEE Transactions on Circuits and Systems, vol. 33, no. 11, pp. 1072-1118, 1986.

[51] D. Matignon, "Stability results for fractional differential equations with applications to control processing," Сomputational Engineering in Systems Applications, vol. 2, no. 1, pp. 963-968, 1996.

[52] S. Bhalekar and M. Patil, "Singular points in the solution trajectories of fractional order dynamical systems," Chaos: An Interdisciplinary Journal of Nonlinear Science, vol. 28, no. 11, Article ID 113123, 2018.

[53] J. L. Echenausía-Monroy, G. Huerta-Cuellar, R. JaimesReátegui, J. H. García-López et al., "Multistability emergence through fractional-order-derivatives in a PWL multi-scroll system," Electronics, vol. 9, no. 6, p. 880, 2020.

[54] J. L. Echenausía-Monroy, H. E. Gilardi-Velázquez, R. JaimesReátegui, V. Aboites, and G. Huerta-Cuellar, "A physical interpretation of fractional-order-derivatives in a jerk system: electronic approach," Communications in Nonlinear Science and Numerical Simulation, vol. 90, Article ID 105413, 2020.

[55] K. Diethelm, N. J. Ford, and A. D. Freed, "A predictor-corrector approach for the numerical solution of fractional differential equations," Nonlinear Dynamics, vol. 29, no. 1, pp. 3-22, 2002. 